\title{
Rapid Detection and Subsequent Isolation of Bioactive Constituents of Crude Plant Extracts
}

\author{
K. Hostettmann ${ }^{1,2}$, J.-L. Wolfender ${ }^{1}$, and S. Rodriguez ${ }^{1}$ \\ ${ }^{1}$ Institut de Pharmacognosie et Phytochimie, Université de Lausanne, BEP, CH-1015 Lausanne, Switzerland \\ 2 Address for correspondence
}

Received: September 4, 1996; accepted: September 21, 1996

\begin{abstract}
Rapid detection of biologically active natural products plays a strategical role in the phytochemical investigation of crude plant extracts. In order to perform an efficient screening of the extracts, both biological assays and HPLC analysis with various detection methods are used. Combined techniques such as HPLC coupled to UV photodiode array detection (LC/UV) and to mass spectrometry (LC/MS or LC/MS/MS) provide useful structural information on the metabolites on-line prior to isolation. The recent introduction of HPLC coupled to nuclear magnetic resonance (LC/NMR) represents a powerful complement to the LC/UV/MS screening. Various plants belonging to the Gentianaceae and Leguminosae families have been analysed by LC/UV, LC/MS, LC/MS/MS, and LC/NMR. The use of all these coupled techniques allows the structural determination of known plant constituents rapidly and with only a minute amount of plant material. With such an approach, the time-consuming isolation of common natural products is avoided and an efficient targeted isolation of compounds presenting interesting chemical or biological features can be performed.
\end{abstract}

Key words: Plant extracts, LC coupled techniques, LC/UV, LC/MS, LC/MS/MS, LC/NMR.

\section{Introduction}

The plant kingdom represents an extraordinary reservoir of novel molecules. Of the estimated $400,000-500,000$ plant species around the globe, only a small percentage has been in- vestigated phytochemically and the fraction submitted to biological or pharmacological screening is even lower. Since plants may contain hundreds, or even thousands, of metabolites, there is currently a resurge of interest in the vegetable kingdom as a possible source of new lead compounds for introduction into therapeutical screening programmes. The rapid disappearance of tropical forests and other important areas of vegetation has meant that it is essential to have access to methods which lead to the rapid isolation and identification of bioactive natural products. The approach adopted to obtain an exploitable pure plant constitutent involves interdisciplinary work in botany, pharmacognosy, pharmacology, chemistry, toxicology and can be formulated as follows (Fig. 1)(1):

- selection, collection, botanical identification, and preparation of plant material;

- extraction with suitable solvents and preliminary analysis;

- biological, pharmacological and chemical screenings (LC/UV/ MS) of crude extracts;

- chromatographic separation of pure bioactive constituents guided by bioassays;

- structure determination;

- analysis and pharmacological profile of pure compounds and toxicological testing;

- partial or total synthesis and preparation of derivatives for structure-activity relationships.

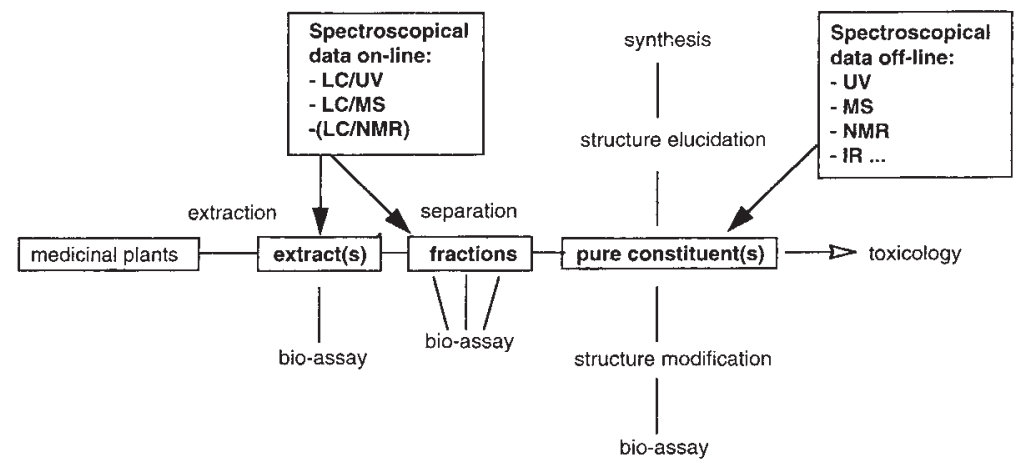

Planta Medica 63 (1997) 2-10

() Georg Thieme Verlag Stuttgart · New York
Fig. 1 Procedure for obtaining the active principles from plants and use of LC-coupled techniques as strategic analytical screening tools during the isolation process of a plant extract. 


\section{Biological Screening of Crude Extracts}

Crucial to any investigation of plants with biological activities is the availability of suitable bioassays for monitoring the required effects (2). In order to cope with the number of extracts and fractions from bioactivity-guided fractionation steps, the capacity for high sample throughput is necessary. The test systems should ideally be simple, rapid, reproducible, and inexpensive. If active principles are only present at low concentrations in the crude extracts, the bioassays have to be sensitive enough for their detection. At the same time, the number of false positives should be reduced to a minimum. When deciding which bioassays to employ in research on plant constituents, the first step is to choose suitable target organisms. These can be lower organisms (microorganisms, insects, crustaceans, molluscs), isolated subcellular systems (enzymes, receptors, organelles), cultured cells of human or animal origin, isolated organs of vertebrates, or whole animals. However, the right target has to be found for the disease in question.

The complexity of the bioassay has to be designed as a function of the facilities, resources, and personnel available. Most phytochemical laboratories engaged in the investigation of bioactive medicinal plants do not have extensive biological test facilities. Consequently, efforts have been made to introduce simple, inexpensive "bench-top" bioassays for the rapid screening of plant extracts and fractions with a variety of targets, ranging from a test for general toxicity with brine shrimp to snail killing activity with Biomphalaria glabrata. In this way, different properties and types of ailment, including microbial afflictions and parasitic diseases, can be investigated.

\section{Chemical Screening with LC-Coupled Techniques}

As the number of targets for biological screening is limited, an efficient system for the chemical screening of the extracts is thus also needed in order to detect new leads which can be potentially interesting from a chemical view point. Early recognition of plant metabolites, at the earliest stage of separation as possible, is also essential in order to avoid a time-consuming isolation of common constituents. In our laboratory LC-coupled techniques have been mainly used for the chemical screening of crude plant extracts.

\section{Coupling in HPLC}

High performance liquid chromatography (HPLC) is used routinely in phytochemistry to "pilot" the preparative isolation of natural products (optimisation of the experimental conditions, checking of the different fractions throughout the separation) and to control the final purity of the isolated compounds (3). For chemotaxonomic purposes, the botanical relationships between different species can be shown by chromatographic comparison of their chemical composition. Comparison of chromatograms, used as fingerprints, between authentic samples and unknowns permits identification of drugs and/or search for adulteration. HPLC is thus the most well fitted technique for an efficient separation of crude plant extracts and can be coupled with different spectroscopic detection methods.

HPLC coupled with UV photodiode array detection (LC/UV) has been used since more than a decade by phytochemists for screening extracts (4) and is now widely spread in many laboratories. The UV spectra of natural products give useful information on the type of constituents and also, as it is the case for polyphenols, information on the oxidation pattern. New instruments allow the recording of UV spectra of reference compounds in databases and computer matching can be realised automatically when screening for known constituents.

HPLC coupled to mass spectrometry (LC/MS) has been introduced recently and is still not widely spread in the phytochemical community (5). At present, MS is one of the most sensitive methods of molecular analysis. Moreover, it has the potential to yield information on the molecular weight as well as on the structure of the analytes. Due to its high power of mass separation, very good selectivities can be obtained. The coupling between LC and MS has not been straightforward since the normal operating conditions of a mass spectrometer (high vacuum, high temperatures, gas-phase operation, and low flow rates) are diametrically opposed to those used in high performance liquid chromatography (HPLC), namely liquidphase operation, high pressures, high flow rates, and relatively low temperatures (6). Because of the basic incompatibilities between HPLC and mass spectrometry (MS), on-line coupling of these instrumental techniques has been difficult to achieve and to cope with these problems, different LC/MS interfaces have been developed. Each of these interfaces has its own characteristics and range of applications and several of them are suitable for the analysis of plant secondary metabolites (7). In our appproach to LC/MS, mainly used for the HPLC screening of crude plant extracts, three interfaces, thermospray (TSP) (8), continuous flow FAB (CF-FAB) (9), and electrospray (ES) (10) have been investigated (7). They cover the ionisation of relatively small non-polar products (aglycones, $200 \mathrm{u}$ ) to high polar molecules (glycosides, $2000 \mathrm{u}$ ). LC/TSP-MS allowed a satisfactory ionisation of moderately polar constituents such as polyphenols or terpenoids in the mass range of 200-800 u. For larger polar molecules such as saponins (MW > $800 \mathrm{u}$ ), CF-FAB or ES are the methods of choice (7). In our laboratory, thermospray is the most widely used interface.

HPLC coupled with nuclear magnetic resonance (LC/NMR), despite being known for over fifteen years (11), has not yet been a widely accepted technique, mainly because of its lack of sensitivity. However, the recent progress in pulse field gradients and solvent suppression, the improvement in probe technology and the construction of high field magnets have given a new impulse to this technique. LC/NMR has an important potential for on-line structure identification of natural products. Indeed, nuclear magnetic resonance (NMR) spectroscopy is by far the most powerful spectroscopic technique for obtaining detailed structural information about organic compounds in solution (12). While the LC coupling itself was rather straightforward compared to LC/MS (13), the samples are flowing in a non-rotating glass tube $(60-180 \mu \mathrm{l})$ connected at both ends with HPLC tubing, the main problem of LC/NMR was the difficulty of observing analyte resonances in the presence of the much larger resonances of the mobile phase. This problem was even worsened in the case of typical LC reversed phase operating conditions, where more than one protonated solvent was used and where the resonances changed frequencies during the analysis in gradient mode. Furthermore, the continuous flow of sample in the detector coil complicated solvent suppression. These problems have now been overcome thanks to the development of fast reliable and powerful solvent suppression techniques such as WET (14), which produced high 


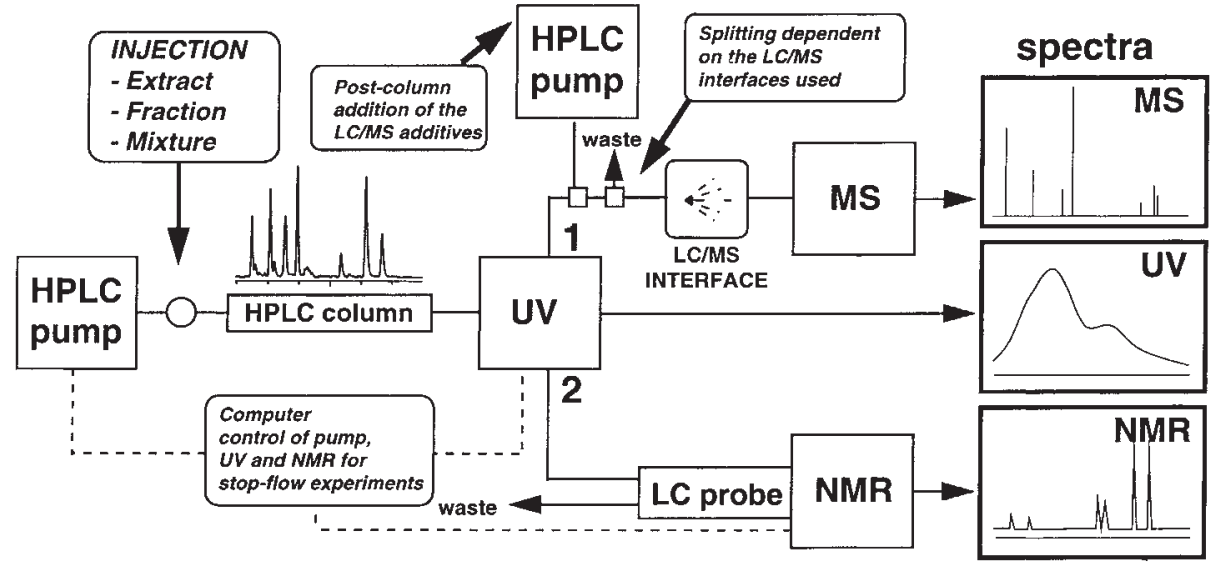

Fig. 2 Schematic representation of the experimental setup used for LC/UV/MS (1) and LC/UV/NMR (2) analyses.

quality spectra in both on-flow and stop-flow modes. These techniques consist of a combination of pulsed field gradients, shaped RF pulses, shifted laminar pulses, and selective ${ }^{13} \mathrm{C}$ decoupling and are much faster than classical presaturation techniques previously used in this field (14). Thus, in reversed HPLC conditions, non-deuterated solvents such as $\mathrm{MeOH}$ or MeCN can be used, while water is replaced by $D_{2} O$.

A general setup of the LC/UV/MS and the LC/NMR configuration used is presented in Fig. 2.

\section{LC/UV/MS in the screening of polyphenols}

When screening plant extracts for UV active compounds such as polyphenols with interesting properties, a multidimensional approach to their chromatographic analysis is of great significance. Indeed, UV spectra recorded on-line give useful complementary information (type of chromophore or pattern of substitution) to those obtained with LC/MS. LC/UV/MS has been used extensively in our laboratories to screen numerous Gentianaceae plants in the search for xanthones, potential new selective and reversible inhibitors of monoamine oxidase (15, 16).

For the LC/UV/MS analyses, a minute amount of the crude extracts of the Gentianaceae (ca. $300 \mu \mathrm{g}$ ) was directly separated on a $4 \mathrm{~mm}$ i.d. reversed-phase column with an acidic acetonitrile-water gradient ( $1 \mathrm{ml} / \mathrm{min}$ ). Under these conditions, it was possible to obtain a satisfactory separation of the main constituents. The UV spectra recorded on-line allowed the distinction of xanthones from the other constituents. These polyphenols show characteristic UV spectra usually with four bands of decreasing intensity between $200-400 \mathrm{~nm}$. The other metabolites were generally secoiridoids (one UV maximum at ca. $240 \mathrm{~nm}$ ) or flavones (two UV maxima at $200-400 \mathrm{~nm}$ ). For the LC/MS analysis of these extracts, the TSP interface which is well adapted for the ionisation of moderately polar molecules (17), was chosen.

For example, the LC/UV/MS analysis of a methanolic root extract of a Gentianaceae from Argentina, Gentianella cabrerae, presented different peaks characteristic of secoiridoids, flavone $C$-glycosides, and xanthones. In order to show the type of information recorded on-line, the LC/TSP-MS and LC/UV spectra of two polyphenols have been selected. The UV spectrum of 2 was characteristic for a xanthone substituted in $1,3,5,8$
(Fig. 3). The LC/TSP-MS of this peak exhibited a protonated molecular $[\mathrm{M}+\mathrm{H}]^{+}$ion at $m / z 275$. The molecular weight of this xanthone was thus $274 \mathrm{u}$. As the mass of a bare xanthone nucleus is $196 \mathrm{u}$, this indicated that the polyphenol was tetra-
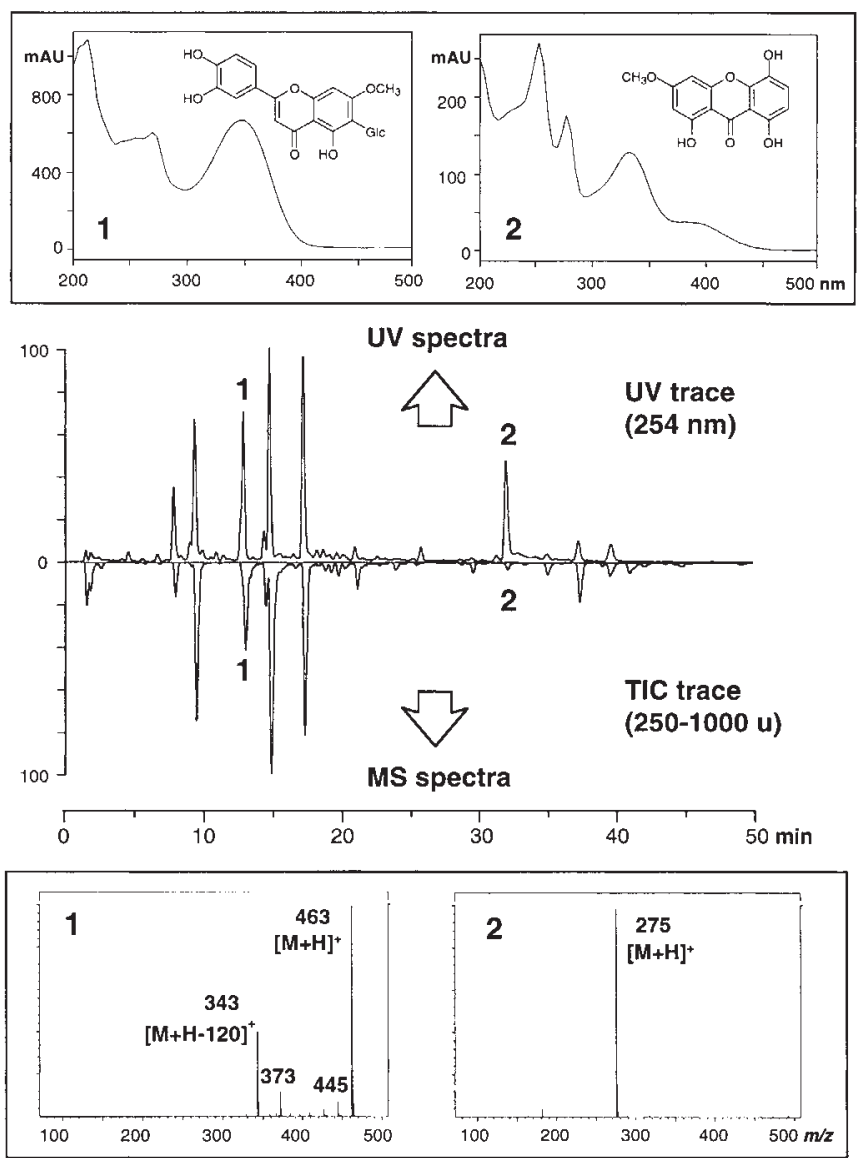

Fig. 3 LC/UV/MS analysis of the methanolic extract of Gentianello cabrerae (Gentianaceae). The UV and TSP-MS of a flavone C-glycoside swertiajaponin (1) and a xanthone bellidifolin (2) are displayed. The UV trace was recorded at $254 \mathrm{~nm}$ and UV spectra from 200 to $500 \mathrm{~nm}$. The LC/TSP-MS trace was recorded from 150-900 u. HPLC: Column, RP-18 NovakPak ( $4 \mu \mathrm{m}, 150 \times 3.9 \mathrm{~mm}$ i.d.); gradient, $\mathrm{CH}_{3} \mathrm{CN}-\mathrm{H}_{2} \mathrm{O}(0.1 \%$ TFA) $5: 95 \rightarrow 65: 35$ in $50 \mathrm{~min}(1 \mathrm{ml} / \mathrm{min}), 0.5 \mathrm{M}$ $\mathrm{NH}_{4} \mathrm{OAC}(0.2 \mathrm{ml} / \mathrm{min}$ post-column). TSP: positive ion mode; filament off; vaporiser $100^{\circ} \mathrm{C}$; source $280^{\circ} \mathrm{C}$. 
substituted by three hydroxy $(3 \times 16 \mathrm{u})$ and one methoxy groups $(1 \times 30 \mathrm{u})$. A comparison of the on-line UV spectra with those of tetrasubstituted xanthones from an in-house UV database confirmed that this compound was indeed bellidifolin, a widespread xanthone in the genus Gentianella. Compound 1 presented a UV spectrum characteristic for a flavonoid compound. Its LC/TSP-MS was more complicated than that recorded for 2 and showed different fragments. Compound 1 exhibited a protonated molecular ion $[\mathrm{M}+\mathrm{H}]^{+}$at $m / z$ 463 and fragments $[\mathrm{M}+\mathrm{H}-90]^{+}$and $[\mathrm{M}+\mathrm{H}-120]^{+}$at $\mathrm{m} / \mathrm{z} 373$ and 343 which are characteristic for the cleavage of flavone $C$ glycosides. According to these data, $\mathbf{1}$ was most probably a flavone C-glycoside substituted by three hydroxy and one methoxy groups. Two isomeric flavones occurring in Gentianaceae, isoscoparin and swertiajaponin, fitted with such data. An LC/TSP-MS/MS (see below) was performed in order to identify it fully. Following the same approach, the other constituents of this extract were also identified on-line.

The LC/UV chromatogram of the dichloromethane extract of another Gentianaceae, Halenia corniculata from Mongolia, presented more than 19 peaks having UV spectra characteristic for xanthones (16). Two types of xanthone $O$-glycosides as well as xanthone aglycones were detected in this extract. As LC/MS can also be used as a very selective detector, a display of the selective ion traces of the molecular ions of the aglycones and glycosides of these xanthones was used for their specific detection. As shown in Figure $\mathbf{4}$ for example, three xanthones of H. corniculata, 3, 4 and 5, appeared in the single ion trace $363 \mathrm{u}$, which indicated the presence of a common pentasubstituted aglycone (one hydroxy and five methoxy groups) in each case. This was confirmed by similar UV spectra for all the three xanthones. The glycosides $\mathbf{3}$ and $\mathbf{4}$ were detected separately at 709 and $679 \mathrm{u}$, respectively. These ions corresponded to weak molecular sodium adducts $[\mathrm{M}+\mathrm{Na}]^{+}$of the xanthone glycosides. Important fragments corresponding to the aglycone moiety $[\mathrm{A}+\mathrm{H}]^{+}$were also observed in the LC/TSP-MS (Fig. 4). The difference of $316 u$ between the ions $[\mathrm{M}+\mathrm{Na}]^{+}$and $[\mathrm{A}+\mathrm{H}]^{+}$ in the TSP-MS of 4 was characteristic of the loss of a di- saccharide moiety constituted of hexosyl and pentosyl units [(-pentosyl (132) -hexosyl (162) $-\mathrm{Na}(23)+\mathrm{H}(1)]$. Likewise, a difference of $346 \mathrm{u}$ [(-hexosyl (162) - hexosyl (162) - Na (23) $+H(1)]$ for 3 indicated the presence of a dihexosyl derivative. As primeverosyl and gentiobiosyl are the only disaccharide residues corresponding to these masses which have been found to date in the Gentianaceae family, $\mathbf{3}$ and $\mathbf{4}$ were respectively the gentiobiosyl and the primeverosyl derivatives of the corresponding free aglycone $\mathbf{5}$. The UV spectra of the three compounds were very similar but did not correspond to any of the available data. Thus, a rare oxidation pattern was indicated by these on-line data. Following this approach, other series of aglycones and corresponding glycosides $(\mathbf{6}-\mathbf{1 9})$ were detected on-line (Fig. 5). The isolation of the most interesting xanthones was subsequently undertaken (16).

The dichloromethane extract of H.corniculata was first fractionated by gel filtration giving twelve fractions. The LC/UV analysis of these fractions allowed the localisation of the xanthone glycosides of interest in fraction 2, which was subjected to MPLC separation on a reversed phase column in two batches (Fig. $\mathbf{6}$ ). With the aid of different NMR experiments, the structures of the isolated xanthone glycosides $4,10,11,14,15$, and $\mathbf{1 7}$ were fully assigned. This information together with the on-line data obtained was used to confirm the on-line identification of 11 other xanthones of the extract (Fig. 5). As expected from the on-line data, the xanthones $(\mathbf{3}-\mathbf{7}, \mathbf{9}, 11,16$, and 17) were new natural products (16).

These two examples of LC/UV/MS analyses showed that for simple known compounds like xanthones, a precise identification can be performed on-line, provided that some information about the type of the constituents and their occurrence in given plant families is already available.
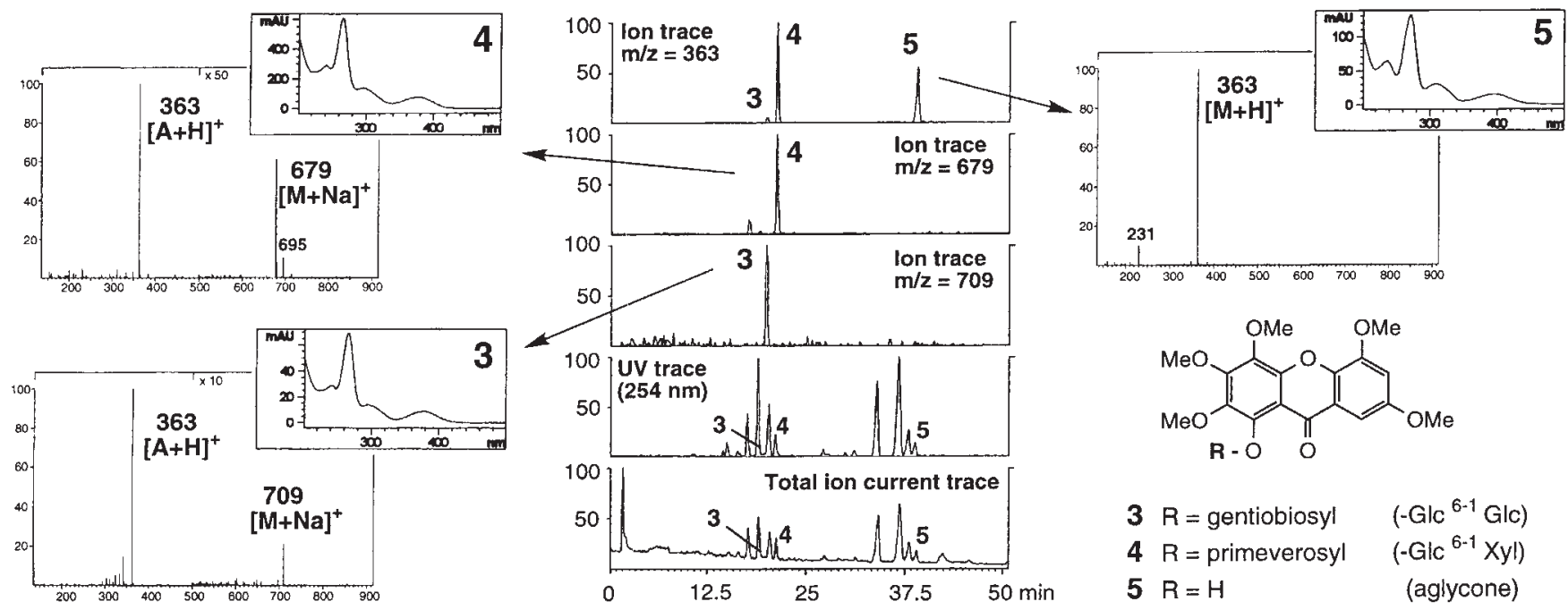

$3 \mathrm{R}=$ gentiobiosyl
$4 \mathrm{R}=$ primeverosyl
$5 \mathrm{R}=\mathrm{H}$

$\left(-\mathrm{Glc}^{6-1} \mathrm{Glc}\right)$

$\left(-\right.$ Glc $\left.^{6-1} \mathrm{Xyl}\right)$

(aglycone)

Fig.4 LC/UV/MS analysis of the crude dichloromethane extract of Halenia corniculata. The UV and MS of three related xanthones (aglycone 5 and its two glycoside derivatives 3 and 4) are displayed. The ion $\mathrm{m} / z 363$ is the main fragment and corresponds to the aglycone moiety [A $+\mathrm{H}]^{+}$. The ion traces displayed at $\mathrm{m} / z 679$ and 709 correspond to the molecular adduct ions $[\mathrm{M}+\mathrm{Na}]^{+}$of gentiobioside 3 and primeveroside 4 , respectively. Same conditions as in Fig. 3. 

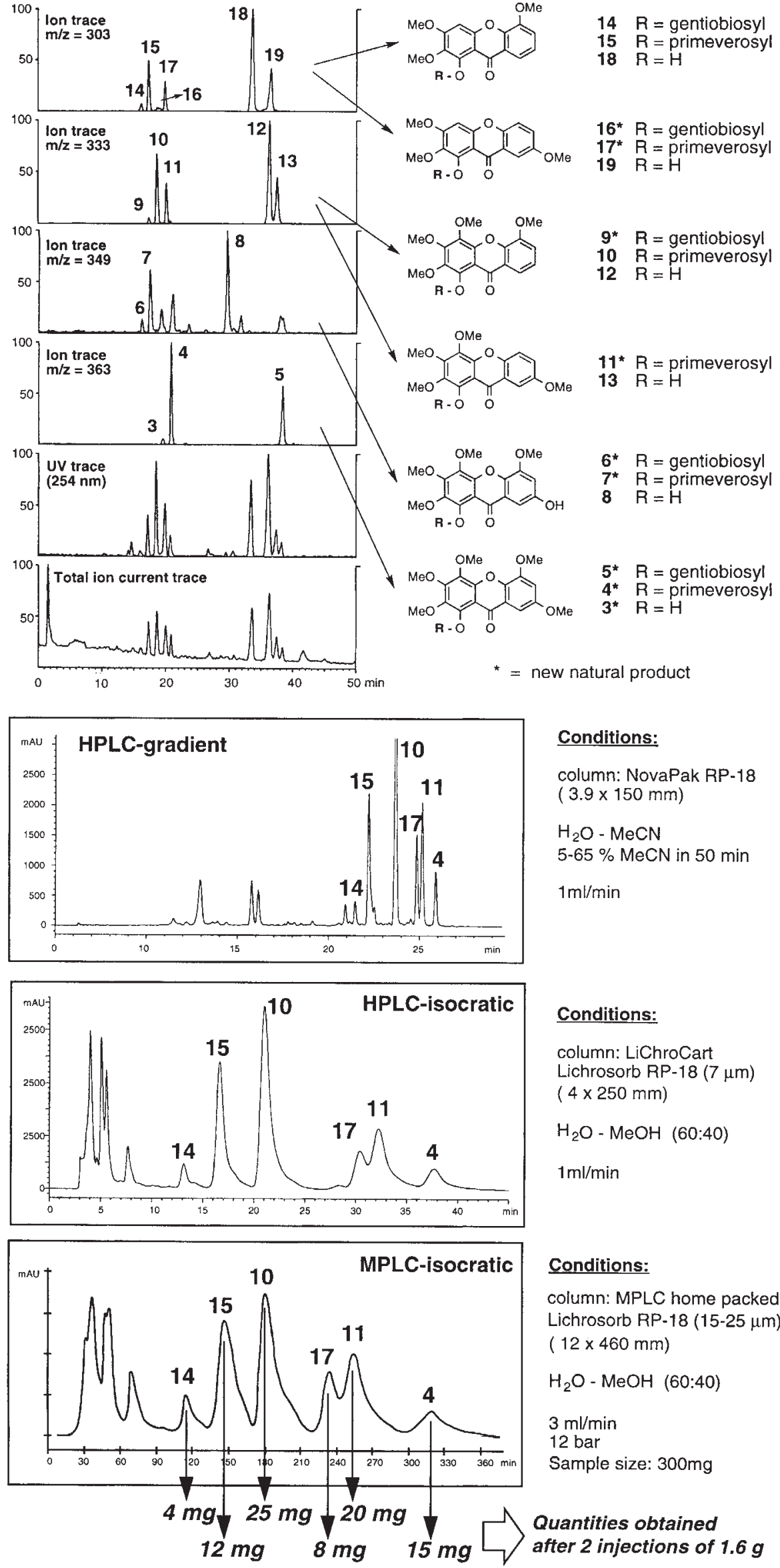

\section{Conditions:}

column: MPLC home packed Lichrosorb RP-18 $(15-25 \mu \mathrm{m})$ $(12 \times 460 \mathrm{~mm})$

$\mathrm{H}_{2} \mathrm{O}-\mathrm{MeOH}(60: 40)$

$3 \mathrm{ml} / \mathrm{min}$

12 bar

Sample size: $300 \mathrm{mg}$
Fig.5 LC/UV/MS analysis of the crude dichloromethane extract and structures of all the xanthones identified in Holenia corniculato. For each oxidation pattern, the aglycone moiety ion trace is displayed. Thus, series of xanthones with the same aglycone can be observed. Same conditions as in Fig. 3.
Fig. 6 Isolation of xanthones from the dichloromethane extract of Halenia corniculata. HPLC-MPLC transposition for the separation of the xanthone glycosides of fraction $3(3.3 \mathrm{~g})$ obained after gel filtration of the crude extract on Sephadex LH-20. 
<smiles>COc1cc2oc(-c3ccc(O)c(O)c3)cc(=O)c2c(O)c1C(O)COC1C(C)C(O)C(O)C1CO</smiles><smiles>COc1cc2oc(-c3ccc(O)c(O)c3)cc(=O)c2c(O)c1/C=C/[OH2+]</smiles>

LC/TSP-MS/MS of $[\mathrm{M}+\mathrm{H}-120]+$ of 1

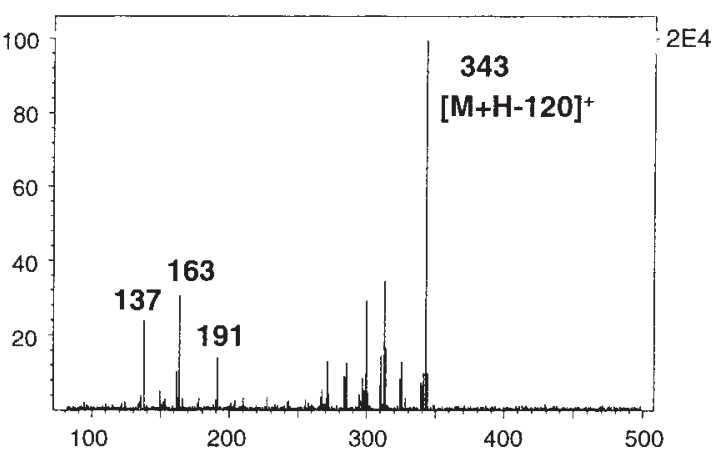

Fig. 7 LC/TSP-MS/MS obtained on-line for swertiajaponin (1) in the methanolic extract of Gentianella cabrerae. The LC/TSP-MS/MS of the ion $[\mathrm{M}+\mathrm{H}-120]^{+}$at $\mathrm{m} / \mathrm{z} 363$ exhibited characteristic fragments for the substitution of the B-ring and for the glycosidation at C-6.

\section{LC/MS/MS for more detailed on-line structural identification}

The LC/MS interfaces generally produce a soft ionisation of the plant metabolites and gave mainly molecular weight information (7). Some structurally significant fragments such as sugar losses can be observed, with LC/TSP-MS for example, but usually only limited structural information is obtained. In order to record more characteristic fragments, collision induced dissociation (CID) spectra of the metabolites can be performed using tandem mass spectrometers (MS/MS).

For example, LC/TSP-MS/MS has been used successfully for the on-line characterisation of flavone $C$-glycosides. As shown previously in Fig. 3, the LC/UV/MS analysis of G. cabrerae revealed the presence of different flavonoid $C$-glycoside constituents (peaks eluting between 12-15 min, Fig. 3). The LC/TSP-MS of one of them (1) is shown in Fig. 3. As mentioned, the LC/TSPMS data only were not sufficient enough to determine if $\mathbf{1}$ was isoscoparin or swertiajaponin, two isomeric flavone $C$-glycosides having a methoxy group either at position C-3' or at C-7, respectively. In order to attain more information on the distribution of the substituents of the A- and B-rings of this compound, an LC/TSP-MS/MS of 1 was carried out by chosing the intense $[\mathrm{M}+\mathrm{H}-120]^{+}$ion as parent ion (Fig. 7). The experiment was performed on a triple quadrupole instrument, in which the first quadrupole was set in order to filter the ion $[M+$ $\mathrm{H}-120]^{+}$. This ion was then selectively fragmented in the collision chamber (the 2 nd quadrupole) containing argon by collision induced dissociation (CID). Finally, the spectrum was recorded by scanning the 3rd quadrupole. In this case, a classic fragmentation of the aglycone moiety of the flavonoid gave an ion at $m / z 137$. This fragment was indicative of a B-ring having two hydroxy groups, confirming the localisation of the methoxy group of 1 on the A-ring. Ions at $m / z 163$ and 191 were specific for flavone $\mathrm{C}$-glycosides having their saccharide moiety at position C-6 and permitted a clear differentiation with 8-C-glycoside flavones which did not present this type of fragmentation (18). These structurally important ions, not observable in the genuine LC/TSP-MS spectrum (see Fig. 3), permitted a partial structural determination of $\mathbf{1}$ as swertiajaponin, a well known flavonoid C-glycoside already found in different Gentiana and Gentianella species.

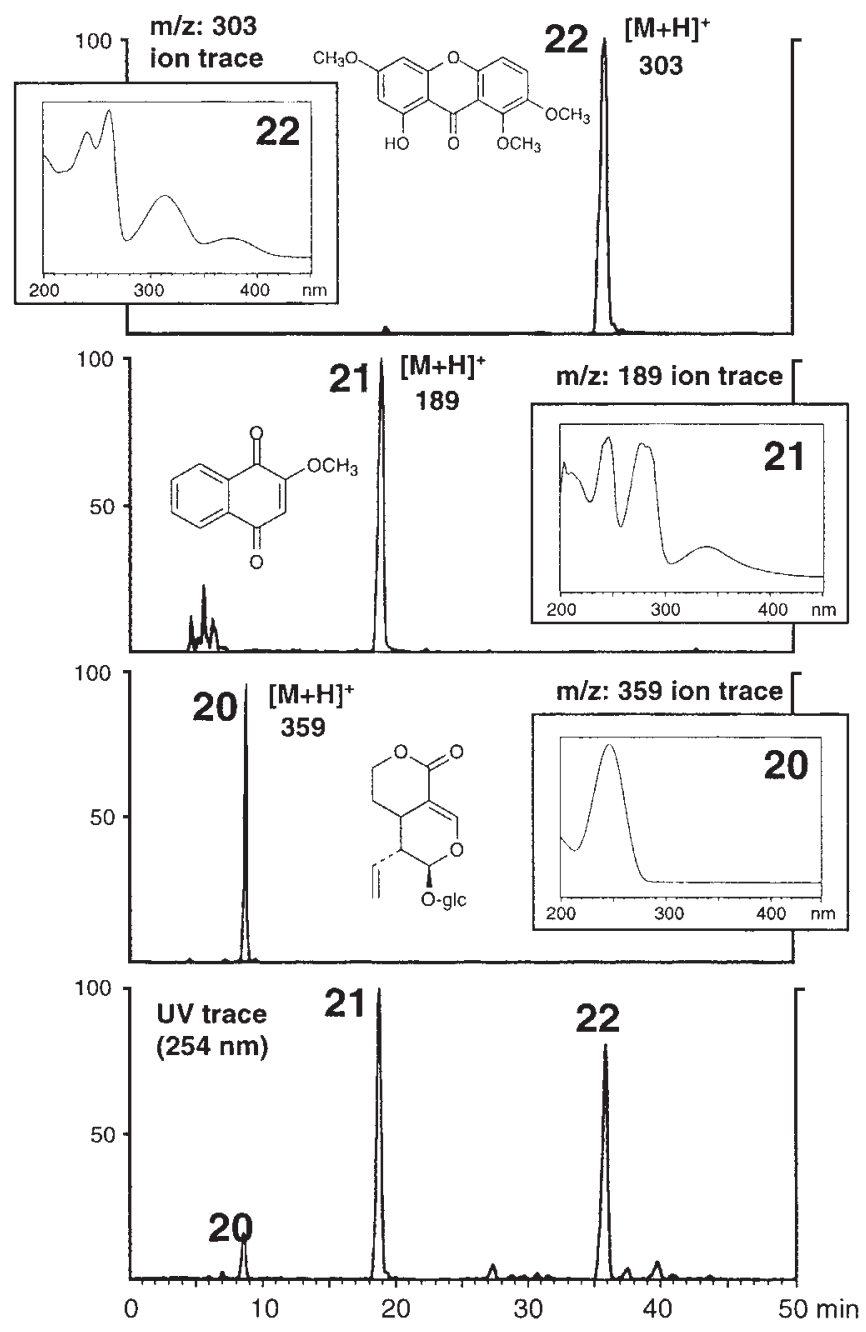

Fig. 8 LC/UV and LC/TSP-MS analysis of the crude $\mathrm{CH}_{2} \mathrm{Cl}_{2}$ extract of Swertia calycina. For each major peak, the single ion LC/MS traces of the protonated molecular ions $[\mathrm{M}+\mathrm{H}]^{+}$were displayed, together with the UV spectra obtained on-line. 


\section{LC/UV/MS and LC/NMR}

The dichloromethane extract of another plant from the Gentianaceae family, Swertia calycina, presented a strong antifungal activity against Cladosporium cucumerinum and Candida albicans. According to the TLC assay, this activity was linked to a strong UV visible spot $\left(\mathrm{R}_{\mathrm{f}}=0.38\right.$; petroleum ether-ethyl acetate; $1: 1)$. As some xanthones are known to be antifungal agents (19), one of these could be the active principle. In order to identify the antifungal agent, the extract has been studied by LC/UV, LC/MS and LC/NMR.

The LC/UV chromatogram of dichloromethane extract of $S$. calycina was more simple than that of the methanolic extract of $\mathrm{H}$.corniculata shown previously and three main peaks (20-23) were detected (Fig. 8). The LC/UV and LC/MS data, together with chemotaxonomical considerations, allowed the identification of most xanthones and secoiridoids of this plant. Compound 22 presented a UV spectrum with four absorption bands characteristic of a xanthone. Its TSP-MS exhibited a strong protonated ion at $303 \mathrm{u}$, indicating a xanthone of molecular weight of 302 , thus substituted by one hydroxy and three methoxy groups. This information, together with the comparison with an in-house UV spectral library, permitted the identification of $\mathbf{2 2}$ as decussatin, a widespread xanthone in the Gentianaceae family. The on-line data obtained for compound $\mathbf{2 0}$ indicated the presence of a secoiridoid type molecule with a molecular weight of $358 \mathrm{u}$. The loss of $162 \mathrm{u}$ observable in the TSP-MS was characteristic for the presence of a hexosyl moiety. These data suggested strongly that $\mathbf{2 0}$ was most probably sweroside, a well-known secoiridoid of the family. The UV spectrum of 21, however, was not attributable to a common polyphenol of the Gentianaceae such as flavones or xanthones. It was very weakly ionised in LC/TSP-MS (see TSP-MS in Fig. 10), but a protonated molecular ion was nevertheless found at $m / z 189$. This small molecular weight (188 u) and the UV spectrum suggested that 21 could be a quinonic compound, but as no metabolite of this type was previously found in the Gentianaceae family, it was not possible to identify it on-line.
In order to confirm these attributions and to obtain more structure information on-line, the same extract of S.calycina was submitted to an on-line $\mathrm{LC} /{ }^{1} \mathrm{H}-\mathrm{NMR}$ analysis on a $500 \mathrm{MHz}$ instrument (20). The same LC conditions as for the LC/UV/MS analysis were used except that the water of the LC gradient system was replaced by $\mathrm{D}_{2} \mathrm{O}$. However, the quantity of extract injected onto the column was increased to $1 \mathrm{mg}$ to obtain at least $20 \mu \mathrm{g}$ for each peak of interest. For the suppression of the solvent signal of $\mathrm{MeCN}$ and its two ${ }^{13} \mathrm{C}$ satellites, as well as the residual HOD peak, a fast sequence called WET was run before each acquisition (20).

Under these conditions, the on-line LC/NMR analysis of S. calycina provided ${ }^{1} \mathrm{H}-\mathrm{NMR}$ spectra for all the major constituents. A plot of the retention time ( $y$ axis) versus the NMR shifts ( $x$ axis) permitted the localisation of the resonances of compounds 20, 21, and 22 (Fig. 9). On this unusual twodimensional chromatogram, strong signals of aromatic methoxy groups were observed around $\mathbf{4}$ ppm for $\mathbf{2 1}$ and 22. Xanthone $\mathbf{2 2}$ exhibited two pairs of aromatic protons, while the quinonic compound 21 presented five other low field protons. The more polar secoiridoid $\mathbf{2 0}$ showed different signals between 3 and $6 \mathrm{ppm}$. The important trace starting from $4.8 \mathrm{ppm}$ (at $0 \mathrm{~min}$ ) and ending to $4 \mathrm{ppm}$ (at $30 \mathrm{~min}$ ) was due to the change of the chemical shift of the residual negative water (HOD) signal during the LC gradient. The traces between 1 and $2.6 \mathrm{ppm}$ were due to residual $\mathrm{MeCN}$ signal and solvent impurities.

A slicing of this bidimensional plot in single on-line $\mathrm{LC} /{ }^{1} \mathrm{H}-\mathrm{NMR}$ spectra for each constituent allowed a precise assignment of their specific resonances. The ${ }^{1} \mathrm{H}-\mathrm{NMR}$ data of $\mathbf{2 0}$ and 22 confirmed their on-line identification by LC/UV/MS as sweroside and decussatin, respectively. On the LC/ ${ }^{1} \mathrm{H}-\mathrm{NMR}$ spectrum of 21 (Fig. 10), two signals ( $2 \mathrm{H}, \delta 8.11, m, \mathrm{H}-5,8$ and $2 \mathrm{H}, \delta 7.89, \mathrm{~m}$, $H-6,7)$ were characteristic of four adjacent protons of an aromatic ring with two equivalent substitutents. The low field shift of the H-5,8 signal indicated that these two protons were in a peri position of the carbonyl functions suggesting most

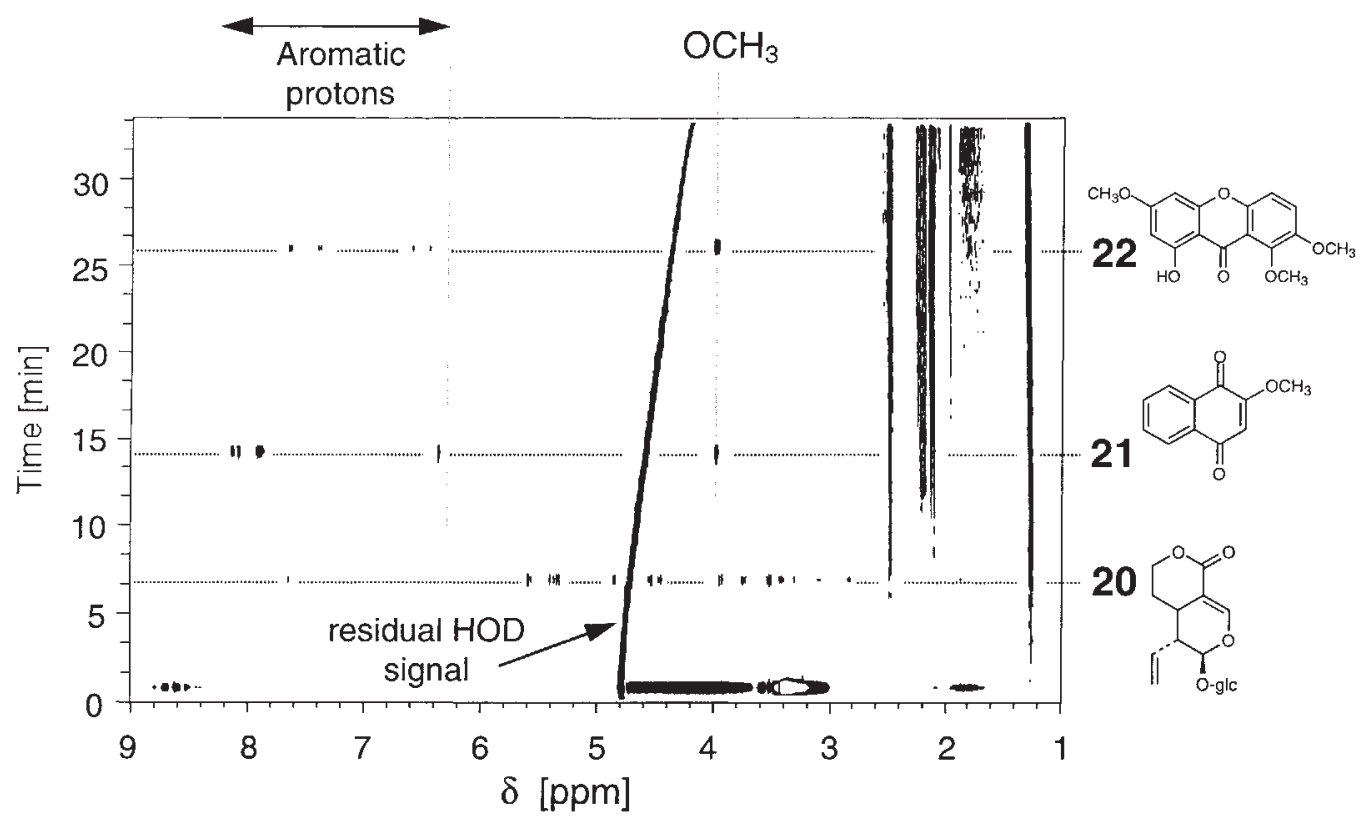

Fig. 9 Bidimensional $L C$ 'H-NMR chromatogram of the crude dichloromethane extract of Swertia calycina. Methoxy groups and aromatic proton signals of $\mathbf{2 1}$ and $\mathbf{2 2}$ are clearly visible together with all the resonances of the monoterpene glycoside 20 . The signal of HOD is negative and was continually shifted during the $L C$ gradient. 


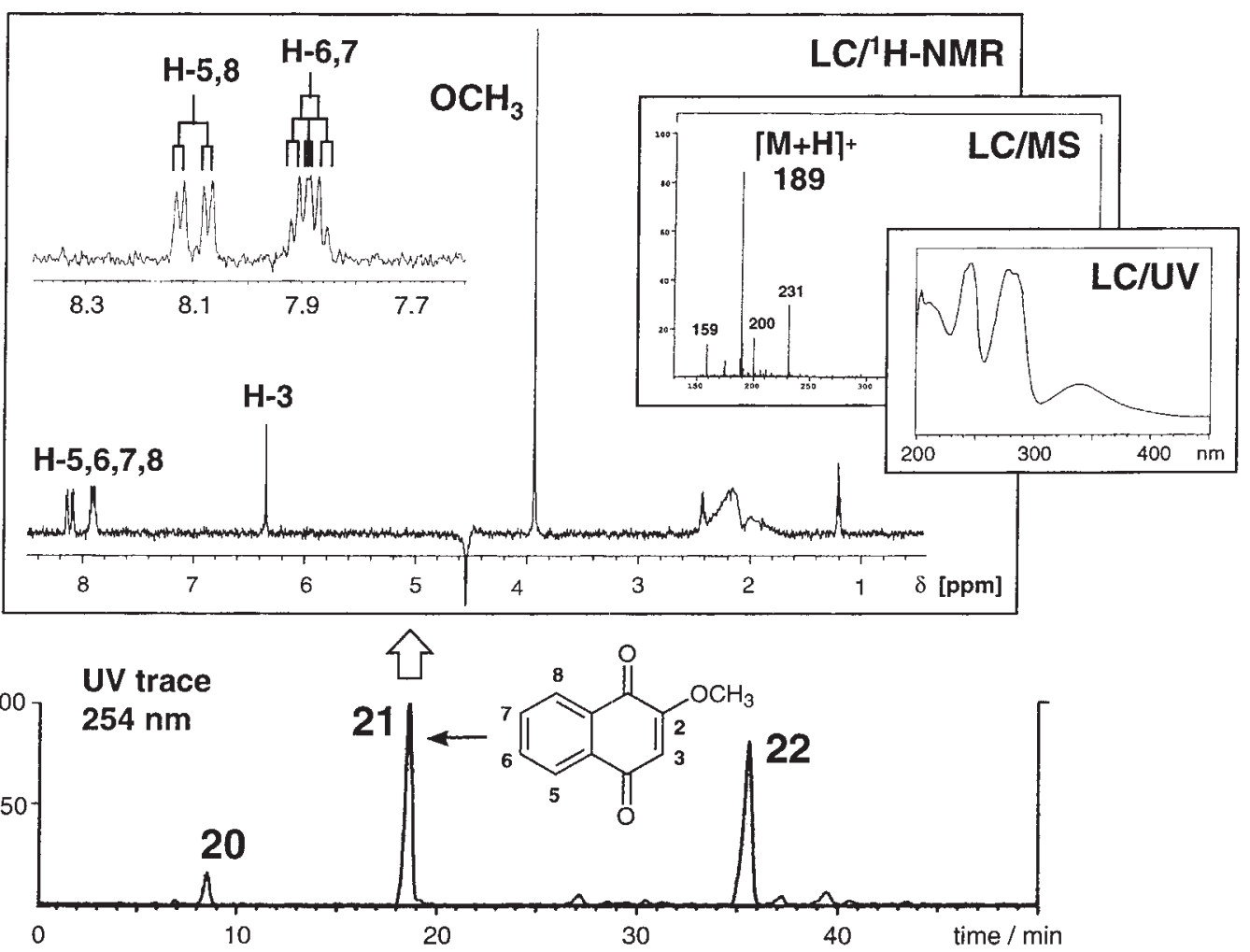

Fig. 10 Summary of all the spectroscopic data obtained on-line for the naphthoquinone 21 in the dichloromethane extract of Swertia calycina.

probably the presence of a naphthoquinone nucleus. The strong bands recorded in UV at $243,248,277,330 \mathrm{~nm}$ confirmed this deduction. The singlet at $4.35 \mathrm{ppm}$ was attributed to $\mathrm{H}-3$ and the remaining methoxy group was thus at position $\mathrm{C}-2$. With this on-line data and the molecular weight deduced from the LC/TSP-MS (MW 188), 21 was finally identified as 2methoxy-1,4-naphthoquinone (Fig. 10). As this was the first naphthoquinone to be reported in Gentianaceae, 21 was specifically isolated, by a combination of column chromatography on silica gel and MPLC on reversed phase, and was found to be the compound responsible for the strong antifungal activity of the extract of S. calycina (21).

This example showed that LC/NMR is an excellent complement to $\mathrm{LC} / \mathrm{UV} / \mathrm{MS}$ and that the combined use of these coupled techniques allows the structure identification of the main constituents of an extract on-line.

\section{Conclusions}

Today, the work of phytochemists lies mainly on bioassayguided fractionation schemes of crude plant extracts. This type of approach has led to the isolation of numerous compounds with interesting activities. Plants contain thousands of constituents with considerably different biological properties. Obviously for any successful investigation of biological material with such a wide range of properties, the future lies in the ability to have as large a number of biological screens as possible. It is also important to take into account the valuable contribution provided by traditional medicine. There are however many obstacles which seriously hamper this type of investigation. First, it is possible that a broad range of structurally diverse compounds contributes to the overall pharmacological activitv may exist. Secondly, there is an urgent need for more appropriate pharmacological models. Existing assays are quite often not reliably predictive for clinical efficiency.

Chemical screening of crude plant extracts, which allows the localisation and efficient targeted isolation of new types of constituents with potential activities, can be considered as an efficient complementary approach to bioassay-guided fractionation. As shown, the addition of highly sensitive, coupled techniques such as LC/UV, LC/MS, and LC/NMR enables a rapid initial screening of crude plant extracts, providing a great deal of preliminary information about the content and nature of constituents of these extracts. According to this structural information, once the novelty or utility of a given constituent is established, it is then important to process the plant extracts in the usual manner, to obtain samples for full structure elucidation and biological or pharmacological testing. In such a way, the unnecessary isolation of common compounds of lower interest is avoided.

The examples discussed have shown that the LC/UV/MS and LC/MS/MS approach alone permits the identification of small metabolites such as xanthones or flavones, provided that some information about the chemotaxonomy of the plant families of interest is already available. For screening more complicated constituent or unknowns, LC/NMR data are of crucial importance for a deeper structural investigation. The recent introduction of $\mathrm{LC} / \mathrm{NMR}$ for the crude plant extract screening will probably make another breakthrough in the on-line structural determination of natural products.

The chemical screening of extracts with such elaborate hynhenated terhninues generates a huge amount of information 
high sample throughput, the next challenge will be to find a way to centralise all these data for rapid pattern recognition by reference to standard compounds. With such an analytical system, phytochemists will then be able to concentrate their efforts on finding new biological targets. This aspect still remains the more difficult problem to solve when searching for new leads.

\section{Acknowledgements}

Financial support was provided by the Swiss National Science Foundation. Thanks are due to Dr. Wolf Hiller, Varian AG, Darmstadt, for the LC/NMR measurements and to Dr. Winfried Wagner-Redeker, Spectronex AG, Basel, for the LC/ES-MS experiments.

\section{References}

1 Hamburger, M., Hostettmann, K. (1991) Phytochemistry 30, 3864 3874 .

${ }^{2}$ Hostettmann, K., Marston, A., Wolfender, J.-L. (1995) Strategy in the search for new biologically active plant constituents, in: Phytochemistry of Plants Used in Traditional Medicine, (Hostettmann, K., Marston, M., Maillard, M., Hamburger, M., eds. ), Oxford Science Publications, Oxford, pp. 17-45.

3 Kingston, D. G. I. (1979) J. Nat. Prod. 42, 237-260.

4 Hostettmann, K., Domon, B., Schaufelberger, D., Hostettmann, M. (1984) J. Chromatogr. 283, 137-147.

${ }^{5}$ Wolfender, J.-L., Hostettmann, K. (1995) Applications of liquid chromatography-mass spectrometry to the investigation of medicinal plants, in: Phytochemistry of Medicinal Plants, (Arnason, J. T., Mata, R., Romeo, J., eds. ), Plenum Press, New York, pp. 189-215.

${ }^{6}$ Garteiz, D. A., Vestal, M. L. (1985) LC Mag. 3, 334-346.

7 Wolfender, J.-L., Rodriguez, S., Hostettmann, K., Wagner-Redeker, W. (1995) J. Mass Spectrom. S35-S46.

${ }^{8}$ Blakley, C. R., Vestal, M. L. (1983) Anal. Chem. 55, 750-754.

${ }^{9}$ Caprioli, R. M., Tan, F., Cotrell, J. S. (1986) Anal. Chem. 58, 2949-2954.

${ }^{10}$ Whitehouse, R. C., Dreyer, R. N., Yamashita, M., Fenn, J. B. (1985) Anal. Chem. 57, 675-679.

11 Watanabe, N., Niki, E., Shimizu, S. (1979) Jeol News 15 A, 2 -5.

12 Albert, K. (1995) J. Chromatogr. A 703, 123-147.

${ }^{13}$ Niessen, W. M. A., van der Greef, J. (1992) Liquid Chromatography-Mass Spectrometry, Marcel Dekker Inc. , New York.

14 Smallcombe, S. H., Patt, S. L., Keiffer, P. A. (1995) J. Magn. Reson. Series A 117, 295-303.

15 Wolfender, J. -L., Hostettmann, K. (1993) J. Chromatogr. 647, 191 202.

16 Rodriguez, S., Wolfender, J.-L., Odontuya, G., Purev, O., Hostettmann, K. (1995) Phytochemistry 40, 1265-1272.

17 Wolfender, J.-L., Maillard, M., Hostettmann, K. (1994) Phytochem. Anal. 5, 153-182.

18 Rath, G., Touré, A., Wolfender, J.-L., Hostettmann, K. (1995) Chromatographia 41,332-342.

19 Marston, A., Hamburger, M., Sordat-Diserens, I., Msonthi, J. D., Hostettmann, K. (1993) Phytochemistry 33, 809-812.

${ }^{20}$ Wolfender, J. -L., Rodriguez, S., Hiller, W., Hostettmann, K. (1996) Phytochem. Anal., in press.

${ }^{21}$ Rodriguez, S., Wolfender, J. -L., Hakizamungu, E., Hostettmann, K. (1995) Planta Med. 61,362-364. 